Case Presentation A 70-year-old diabetic man came with complaints of severe left ear pain and left ear discharge for 2 months and headache and facial deviation for 1 week. On examination, he had tenderness over the tragus and pain on pulling the auricle. External auditory canal was edematous and granulation present. Deviation of angle of mouth toward right was noted. Minimal palsy of left side of soft palate was noticed. Computed tomography showed irregular lytic destruction in the left middle ear. We managed the patient with diabetic control, and broad-spectrum antibiotics through oral, intravenous, and topical routes. Patient was symptomatically feeling better.

Discussion Toulmouche in 1838, was the first to report a case of malignant external otitis. In 1968, Chandler described an aggressive clinical behavior, poor treatment outcome, and a high-mortality rate for the patients affected by this disease. MOE is an aggressive infection in the external auditory canal. It is more common in elderly males with uncontrolled diabetics. Imaging, like computed tomography, magnetic resonance, technetium bone scanning, and radioisotope scanning, helps in confirming diagnosis and extend of spread. Treatment is aggressive medical therapy with pseudomonal coverage. Early infections may be treated with an oral fluoroquinolone (ciprofloxacin), except for resistant organisms. More advanced cases may require parenteral antibiotics with diabetes control and regular follow-up. Hyperbaric oxygen therapy has been used as an adjunct to medical therapy. Surgical debridement of nonviable sequestra of bone is also done.

Conclusion The symptoms and line of management vary with stage at which we diagnose. The earlier it is diagnosed the better the prognosis. As it is a bone eating disease of skull base, it has all the dangers involved and thus requires an aggressive and quick treatment.

\section{A0028: Hearing Outcomes following Canal Wall down Mastoidectomies with Tympanoplasty Architha Menon}

Aim To determine the hearing status in post canal wall down mastoidectomies and comparison in hearing outcome following use of PORPs, TORPs and cartilage grafts, or a combination of all.

Objectives To measure the pure tone average values of patients' preoperatively and postoperatively and assess the improvement or decrement. To evaluate the efficacy of various materials used for reconstruction.

Materials and Methods Patients with chronic suppurative otitis media, atticoantral type in a tertiary institute between January 2013 and January 2018, who underwent canal wall down mastoidectomy with tympanoplasty. The study involved comparison of the pure tone audiogram values done as per standard care of treatment of patients who underwent canal wall down mastoidectomy. The efficacy of reconstruction material used was based on the hearing outcomes of the patient. Results were analyzed using appropriate statistical methods. Outcome measured based on the improvement or worsening of hearing parameters following surgery as per the audiogram values.
Result Hearing improvement was noted in significant percentage following canal wall down mastoidectomy. Further details shall be discussed during the presentation.

\section{A0029: Comparison of Microscopic and Endoscopic Myringotomy With or without Grommet \\ Ashish G. Rao}

Aims Otitis media with effusion (OME) is a common middle ear condition in younger age group and if medical line of treatment fails, it is treated surgically by myringotomy which is usually done under microscope. As the use of endoscope in ear surgeries has increased in the past decade, we thought of doing myringotomy under endoscope and comparing its advantages and disadvantages over conventional microscopic procedure.

Materials and Methods Design: A time-bound descriptive nonrandomized study. Setting: tertiary care hospital. Patients: 33 children of age group 3 to 13 years suffering with OME, who failed medical treatment were included in the study. Methods: 18 underwent myringotomy \pm grommet insertion under microscope, and remaining 15 underwent endoscopic procedure.

Results The primary outcome observed was time taken for the procedure which was more in conventional microscope group than that in endoscopic group. Additional observations like visualization of entire tympanic membrane without adjusting patients head and satisfactory clarity of view and depth perception were found to be better in endoscopic group.

Conclusion Less operative time, satisfactory depth perception, satisfactory clarity of field, and visualization of entire tympanic membrane without turning patient's head make myringotomy \pm grommet insertion with endoscope a better alternative than with a conventional procedure.

Keywords otitis media with effusion, myringotomy, microscope endoscope

\section{A0030: Mapping of Pediatric Cochlear Implant Using Neural Response Threshold and Behavioral Observation Audiometry}

Ashvanee Kumar Chaudhary, ${ }^{1}$ Ashutosh Alakh Avinashi, ${ }^{2}$ Vishwambhar Singh, ${ }^{3}$ Rajesh kumar, ${ }^{4}$ Sunil kumar ${ }^{5}$

Department of Otorhinolaryngology, Institute of Medical Sciences, Banaras Hindu University, Varanasi, Uttar Pradesh, India

Aim To measure the correlation between neural/auditory response threshold (NRT/ART) and most comfortable level for behavioral (MCLB), whichever is better for rehabilitations purpose and to calculate the impedance.

Materials and Methods Mapping may be done by various methods. We performed neural/auditory response threshold (NRT/ART) and behavioral observation audiometry (BOA). NRT/ART is the technique by which we measure the response from the auditory nerve using the hi-resolution bionic ear system. Neural response measurement depends on the neural tissue, stimulus used, and recording technique. BOA is used for observing motor response on presenting sound to the implanted child. Most comfortable level for behavioral 